\title{
Ebola in Westafrika: Resümee der letzten 9 Monate
}

\author{
Am 8. August 2014 erklärte die WHO die Ebola-Epidemie \\ zur gesundheitlichen Notlage von internationaler Tragweite. \\ Bis zum 14. September 2014 wurden 4507 bestätigte und \\ wahrscheinliche Ebola-Fälle sowie 2296 Todesfälle aus \\ Westafrika gemeldet. Die tatsächlichen Zahlen sind sicherlich \\ deutlich höher, und damit ist die aktuelle Ebola-Epidemie \\ größer als alle vorherigen zusammen. \\ N Engl J Med 2014; 371: 1481-1495
}

Das „WHO Ebola Response Team“ berichtet über die klinischen und epidemiologischen Charakteristika der aktuellen Epidemie bis zum 14. September 2014. Sie dokumentieren Trends der Seuche und rechnen zu erwartende Fallzahlen hoch, sollten Eindämmungsmaßnahmen nicht verstärkt werden. Klinische und demographische Daten wurden durch Nachverfolgen von Kontaktpersonen und Auswertung von Klinikaufzeichnungen gesammelt. Weitere Informationen lieferten Fallberichte, Daten aus Laboratorien und Aufzeichnungen von Begräbnissen. Detaillierte Informationen waren von 3343 bestätigten Ebolainfektionen und von 667 wahrscheinlichen Fällen verfügbar und flossen in die Auswertung mit ein.
Das mittlere Erkrankungsalter lag bei 32 Jahren; deutliche Unterschiede der Altersverteilung innerhalb der 5 Länder zeigten sich nicht. 60,8\% der Betroffenen waren zwischen 15 und 44 Jahre alt. Männer und Frauen sind gleichhäufig betroffen. Unter Mitarbeitern im Gesundheitswesen in Guinea, Liberia und Sierra Leone wurde über insgesamt 318 Fälle (davon 151 Todesfälle) berichtet. Im Dezember 2013 traten die ersten Ebolafälle in Guéckédou und Macenta auf. Obwohl in den folgenden Wochen die Erkrankungsfälle und betroffene Regionen rasant zunahmen, sind nicht alle Bezirke betroffen. $90 \%$ der bis zum 14. September 2014 bekannt gewordenen Fälle beschränkten sich auf nur 14 Bezirke.

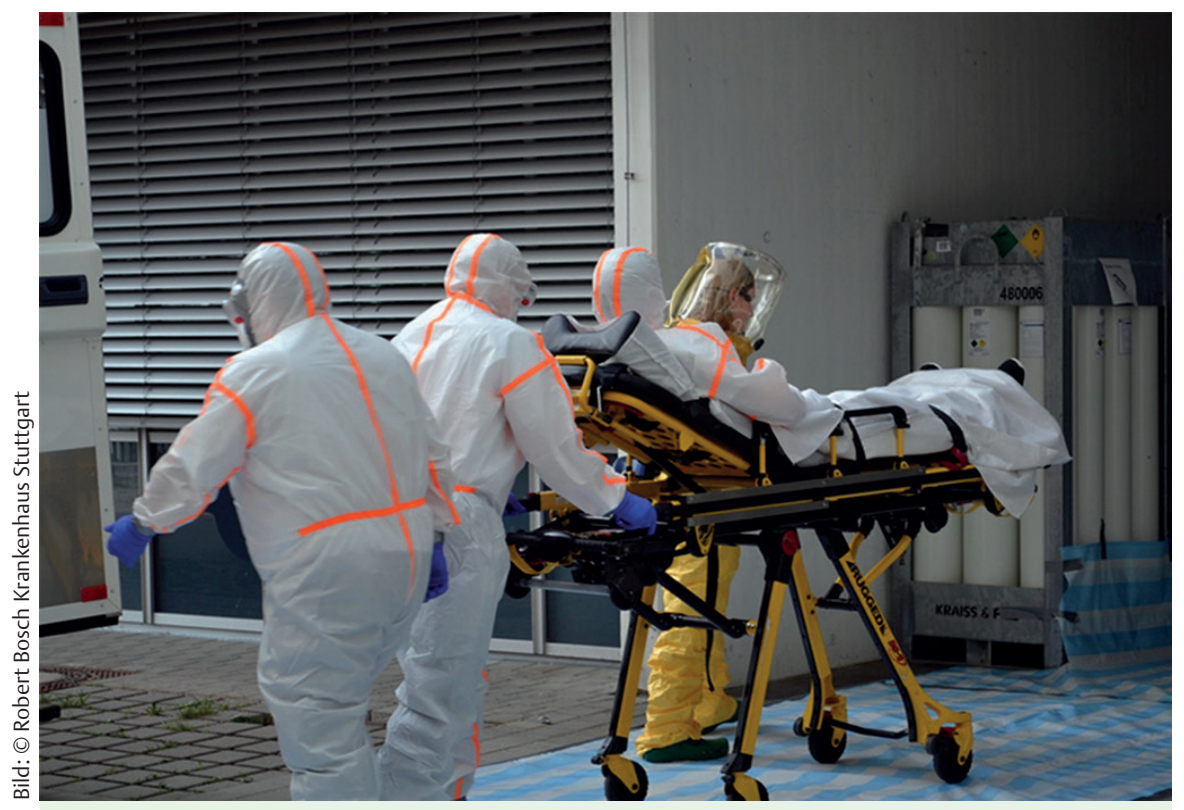

Die Ebola-Epidemie in Westafrika ist von einem nie dagewesenen Ausmaß. Deutsche Kliniken üben regelmäßig den Umgang mit hochansteckenden Patienten.
Die meisten Symptome, die zwischen Beschwerdebeginn und Fallbestätigung berichtet wurden, umfassten Fieber, Abgeschlagenheit, Appetitverlust, Erbrechen, Durchfall, Kopfschmerz und Bauchschmerzen. Hämorrhagische Symptome wurden hingegen nur selten dokumentiert $(<1-5,7 \%)$. Eine „ungeklärte Blutung“" wurde jedoch in $18 \%$ der Fälle berichtet. Bezugnehmend auf die Fälle, über die detailierte Informationen vorlagen, betrug die Todesfallrate in Guinea, Liberia und Sierra Leone 70,8\% (95\%-Konfidenzintervall [KI] 68,6-72,8). Der Verlauf der Infektion einschließlich Symptome, Inkubationszeit (11,4 Tage), Übertragungsintervall (15,3 Tage) ist vergleichbar mit berichteten Daten vorhergehender Ausbrüche und zeigte ebenfalls keine Unterschiede zwischen den einzelnen Ländern.

Auf der Basis des initial exponentiellen Wachstums des Virus beträgt die Basisreproduktionsrate 1,71 (95\%-KI 1,442,01) für Guinea, 1,83 (95\%-KI 1,72-1,94) für Liberia und 2,02 (95\%-KI 1,79-2,26) für Sierra Leone. Die geschätzten aktuellen Reproduktionsraten sind 1,81 (95\%-KI 1,60-2,03) für Guinea, 1,51 (95\%-KI 1,41-1,60) für Liberia und 1,38 (95\%-KI 1,27-1,51) für Sierra Leone. Analog dazu betragen die Verdoppelungszeiten 15,7 Tage (95\%-KI 12,9-20,3) für Guinea, 23,6 Tage (95\%-KI 20,2-28,2) für Liberia und 30,2 Tage (95\%-KI 23,642,3 ) für Sierra Leone. Werden die Eindämmungsmaßnahmen nicht optimiert, liegen die hochgerechneten Fallzahlen für den 2. November 2014 bei 5740 in Guinea, 9890 in Liberia und 5000 in Sierra Leone - insgesamt 20000 Fälle.

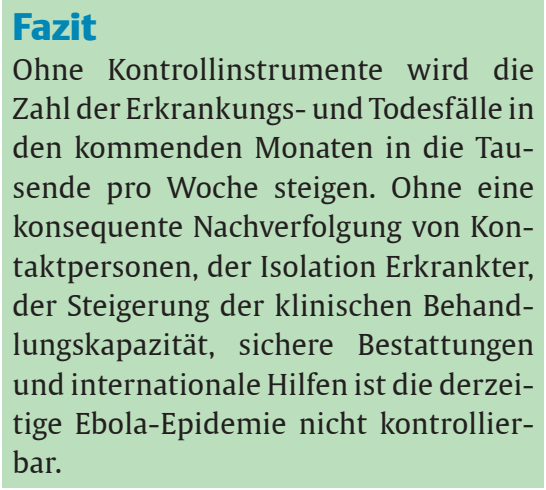

Dr. Markus Escher, Leonberg 